\title{
Reflexiones en torno a las prácticas y características psicosociales de los traba- jadores sexuales masculinos en Bogotá ${ }^{1}$ \\ Reflections on the practices and psychosocial characteristics of male sexual workers in Bogotá
}

\author{
Johan Arturo Barrera Castellanos ${ }^{2}$ \\ Angie Paola Hurtado Roncancio ${ }^{3}$
}

\begin{abstract}
Resumen
El presente artículo es derivado de una investigación cualitativa, enmarcada bajo los planteamientos del construccionismo social, a través de ejercicios de observación participante con diez trabajadores sexuales masculinos, pertenecientes a diversos status en Bogotá, a los cuales se les aplicó una entrevista semi estructurada y un instrumento de caracterización socio-demográfico, esto con el objetivo de comprender cuáles son las prácticas y características psicosociales de estos sujetos sociales. Dejando entrever así dinámicas divergentes que constituyen realidades clandestinas en los sujetos sociales, que se inscriben dentro del servicio sexual masculino.
\end{abstract}

Palabras clave: Trabajo sexual masculino; Invisibilización; Características psicosociales; Prácticas sociales.

\begin{abstract}
This article is derived from a qualitative research, framed under social constructionism approach, throughout participatory observation exercises with ten male sexual workers belonging to different status in Bogota. A semi-structured interview and an instrument of socio-demographic characterization were applied with the aim of comprehending their practices and psychosocial characteristics, showing divergent dynamics that constitute clandestine realities within the social subjects who are inscribed within the masculine sex service.
\end{abstract}

Keywords: Male sexual work; Invisibilization; Social phenomenon; Psychosocial characteristics; Social practices.

\footnotetext{
${ }^{1}$ Artículo de investigación, resultado del proyecto para optar por el título de Trabajadores Sociales bajo el nombre de: Prácticas y características psicosociales de los trabajadores sexuales masculinos. Una ruta de atención de trabajo social desde la perspectiva de la población, realizado en la Universidad Colegio Mayor de Cundinamarca, con el apoyo de la Corporación Red Somos, durante el segundo periodo de 2016 y primer periodo de 2017.

${ }^{2}$ Trabajador Social. Diploma superior en Docencia Universitaria. CLACSO. Miembro del grupo de investigación FÉNIX de la Universidad Nacional Abierta y a Distancia UNAD. https://orcid.org/0000-0002-8843-6057 E-mail: johanbarrera12@gmail.com

${ }^{3}$ Trabajadora Social. Miembro del grupo de investigación FÉNIX de la Universidad Nacional Abierta y a Distancia UNAD. https://orcid.org/0000-0002-1885-275X E-mail: angiepaolah@gmail.com

*Cómo citar este artículo: Hurtado, A., y Barrera, J. (2018). Reflexiones en torno a las prácticas y características psicosociales de los trabajadores sexuales masculinos en Bogotá. Hojas y Hablas, (16), 129-139. DOI: 10.29151/hojasyhablas.n16a9
} 


\section{Introducción}

El Trabajo sexual es una actividad lucrativa, tan antigua como la humanidad misma, Salmerón (2011) plantea que ésta ha pasado y se ha transformado debido a diferentes realidades a lo largo de todas las épocas de la historia, puesto que ésta es reconocida por culturas greco romanas como un servicio reglamentado en el cual se satisfacían y exploraban los placeres del cuerpo en un culto por la figura y expresión de género, que posteriormente es sumergida en posturas prohibicionistas con la expansión del cristianismo vista (en especial a los hombres que la practican) como una práctica que degrada el cuerpo, el espíritu y la sociedad misma. Situación que desde la década de los 70 con los movimientos de liberación sexual y femenina, se trata de ver y abarcar bajo discursos de liberación, autonomía, y toma de decisiones sobre el cuerpo, siempre y cuando no se realicen bajo manipulación u obligación de terceros, dado el caso se hablaría de explotación sexual (vista como en un delito penalizado) (Hernández y Espinosa, 2015). Ésta es casi siempre subordinada bajo la perspectiva, experiencia o cosmovisión de las trabajadoras sexuales femeninas, en una comprensión histórica y geopolítica desde posturas que recaen en la heteronormatividad, teniendo como base constructos sociales que tienden a invisibilizar a los trabajadores sexuales masculinos. (Hernández y Espinosa, 2015).

El termino trabajo sexual, responde por consiguiente a una forma de reivindicación social y apuesta política sobre las personas que ejercen la prostitución, con lo que a través de este sinónimo, se asume y amplía el conglomerado de prácticas derivadas de ésta, percibiéndola como "una profesión como cualquier otra” (Tirado, 2013, p. 82) . Esta visión parte también del hecho de concebir a los sujetos sociales que realizan estas prácticas que trascienden lo económico, como seres consientes y voluntarios dentro de las dinámicas particulares que conglomeran el ejercicio del comercio sexual (Tirado, 2011).

En lo que se refiere a los trabajadores sexu- ales masculinos, se permean diversas dinámicas y realidades, debido a los actuales espectros sociales se han transformado y adaptado para su ejercicio mediante discursos que trascienden en motivaciones que cada persona construye mediante sus vivencias, ya que se evidencia que en el ejercicio de esta actividad, la "concepción del cuerpo como medio para obtener gozo y placer, donde necesariamente prima el dinero como mecanismo de intercambio; pero teniendo una valoración de su propio cuerpo como un símbolo que expresa poder, conquista y dominación." (Tirado, 2005, p. 105), el sujeto tiende a adoptar esta actividad como parte (en algunos casos momentáneo) de su estilo y proyecto de vida, por lo que se "profesionaliza" según etapas, problemáticas y necesidades arraigadas a cada status, (Barrera y Fuquene, 2018) estipulando así un discurso de acuerdo a contextos y tipos de clientes.

Lo anterior hace necesario comprender las prácticas y características psicosociales de los trabajadores sexuales masculinos, desde la mirada de los actores en sus diversos estatus (modalidades tales como calle, estudios de web cam, Scort, masajistas, entre otros) y de este modo logar abarcar, generar claridades que permitan la identificación de los puntos de ruptura en este grupo poblacional.

Este artículo se desarrolla en tres partes: en un primer momento se expone la estructura metodológica utilizada, luego, se exponen los hallazgos y contribuciones según las voces de los actores abordados, para finalmente, exponer las conclusiones constituidas según el ejercicio investigativo realizado.

\section{Metodología}

A partir de ejercicios de observación participante realizados con diez trabajadores sexuales masculinos, se estableció como objetivo lograr comprender cuáles son las prácticas y características psicosociales de estos sujetos sociales. Se constituye este ejercicio investigativo de tipo descriptivo, permitiendo conocer y comprender ciertas 
realidades, motivaciones, espectros y constructos sociales presentes, tanto en la realidad como en el contexto de los trabajadores sexuales masculinos. (Mendicoa, 2003) enmarcándose así bajo el paradigma del construccionismo social, visto desde los planteamientos de Gergen, (2007) como "el estudio socio histórico de las emociones humanas y el metanálisis de la comunicación" ( $p, 8)$ en el que se realiza "un análisis reflexivo de la vida cultural"(p. 296).

Se tuvo como guía, los postulados metodológicos de Bonilla y Rodríguez (1997), a través de un enfoque cualitativo que "implica no abordar el objeto de estudio desde una perspectiva empírica, con hipótesis conceptualmente deductivas, sino que de manera inductiva se pasa del dato observado a identificar los parámetros normativos de comportamiento, que son aceptados por los individuos históricamente identificados" (p. 47), esto a través de un diseño etnográfico que es utilizado como matriz de comprensión de realidades divergentes (Hernández y Espinosa, 2015).

El tipo de muestreo fue aleatorio estratificado, comprendido por una población de diez trabajadores sexuales masculinos, cuyos criterios de inclusión para el estudio fueron el que estos sujetos sociales ejerzan o hayan ejercido desde los diversos status en la ciudad de Bogotá. Estos fueron abordados mediante la construcción de herramientas de recolección de información, tales como la entrevista semi-estructurada y una ficha de caracterización sociodemográfica, con el fin de lograr articular y triangular la información.

\section{Trabajo sexual masculino divergencias de un fenómeno social invisibilizado}

El trabajo sexual masculino en sí mismo se traduce en una serie de realidades de tipo divergentes en el sujeto y su entorno social (Barrera y Fuquene, 2018). Esto en tanto a que el comercio sexual concebido de manera parcial y generalizada, según las características propias del acto sexual, es un ejercicio de toma de decisiones en el que de manera voluntaria se brinda la prestación de un servicio para con el "cliente", a fin de generar un incentivo, ya sea de tipo económico o por especie (Osorio, et al. 2006). Pero en el que no se otorgan ni reconocen las garantías mínimas bajo las cuales en Colombia se considera un trabajo legalmente constituido, tales como afiliación en salud, pensión y cesantías.

\footnotetext{
"Es una ocupación [...] porque esto lo hace cualquiera una profesión es algo más íntegro algo que usted estudia, usted se prepara para ser alguien en la vida[...] mientras usted para ser este... escort usted lo único que tiene es que es querer dinero [...] no tener casi moral (risas) acostarse con cualquiera por dinero" (Entrevista 5, diciembre de 2016, Bogotá.)
}

Ocupación en la que se reconocen riesgos y ventajas en términos de condiciones y espectros sociales, puesto que a diferencia del ejercicio de esta actividad económica por las mujeres, el masculino tiende a estar permeado por estigma, rechazo y discriminación, generando así dinámicas de privacidad y anonimato que van desde el momento, sitio y tipo de encuentro con el cliente, (Tirado, 2013)hasta con el valor mismo del servicio a prestar, ya que dada la normalización y visibilización del trabajo sexual femenino que se genera en espacios más abiertos, ya que son en su mayoría de tipo heteronormativos, pero que en sí mismos conforman una tendencia a brindar servicios a menor costo (en la mayoría de los casos) y en la que el ejercicio de la misma se ve intrínsecamente la reacción carencia- necesidad en el sujeto (Madero, 2012) 
"Las mujeres son más baratas, así de simple, un hombre vale más, muchos hombres es algo más curioso porque una mujer se viene viendo desde hace mucho tiempo, con los hombres se generalizó hace poco, o sea como que es la sociedad hasta ahora cayó en cuenta que también existe la venta sexual masculina" (Entrevista 8, enero de 2017, Bogotá.)

No obstante los, trabajadores sexuales masculinos tienden a establecer dinámicas intrínsecas a sus realidades, que se acoplan a los contextos socioculturales (Zaro, 2006) que determinarían el ejercicio de esta, según las relaciones en el sujeto de actividad económica-placer-libertad-voluntad, que se dan por el deseo del cliente hacia una expresión de género masculina. Por lo que en palabras de Tirado (2005) el ejercicio de esta "tiene que ver con el comercio, pero también y, sobre todo, con la autonomía y el derecho al propio cuerpo, y con la libertad de elegir sobre él de modo personal y autónomo." (p 98), diferenciado así a la explotación sexual, como el hecho de manipulación, u obligación por parte de terceros.

"Es el dinero de otros por placer a la vez, entonces la verdad la explotación sexual tal vez la persona muy probablemente la persona no lo desee si no ya es porque le están obligando a otra que el trabajo sexual es ya porque uno lo elige, uno lo desea porque uno lo busca" (Entrevista 3, diciembre de 2016, Bogotá.)

"Pues sí, sí hay diferencia pues por lo mismo porque para mí el trabajo, la explotación es como algo forzado y el trabajo es como independiente, algo que ya la persona decide y que asume hacer" (Entrevista 4, diciembre de 2016, Bogotá.)

En la que se desprenden una serie de toma de decisiones que permean un estilo de vida (en algunos de los casos), teniendo en cuenta que para el ejercicio de esta el sujeto dedica una cierta cantidad de tiempo, y cumple con ciertas características que según el estatus y lugar se exigen, debido a las dinámicas de competencia dentro del mismo. Por tanto, se estructuran aspectos que el individuo opta por tomar de manera voluntaria y que permean su identidad y su expresión de género masculino, ya que estas se configuran según el tipo de cliente y servicios a ofertar, destinando así recursos económicos y tiempo para perfeccionarlas y que perduren en el tiempo.

"Huyy lo veo pues depende o sea en mi caso yo lo veo como una ocupación porque no me quiero enfocar toda mi vida en ese aspecto, pero puede ser tanto las dos porque hay mucha gente que elige eso como una profesión y se dedican tantas horas, tantos días a cuidar su cuerpo a planear todo su show, por así decirlo, entonces puede ser tanto profesional como ocupacional" (Entrevista 6, diciembre de 2016, Bogotá.)

Características que se establecen intrínsecamente según los espectros sociales establecidos en la expresión de género masculino, a las cuales el cliente evoca en el sujeto, traduciéndose en aspectos físicos, tales como la higiene, tipo de cuerpo, y de vestimenta, entre otros, y de expresión verbal o corporal evidenciándose principalmente por la confianza, seguridad y carisma.(Tirado, 2011) Por tanto, en el ejercicio de la actividad económica, el sujeto de manera voluntaria adopta estas características ya que se ven reflejadas desde el primer contacto con el cliente, hasta la finalización de la prestación del servicio, siendo el estatus y el lugar de ejercicio mecanismos determinantes para ello.

"Uno tiene que ser primero que todo muy aseado, heee tratar de vestirse bien [...] uno tiene que pensar cómo llamarle la atención a un hombre [...] siempre andar sonriendo así Ud. tenga mil problemas en la cabeza Ud. siempre tiene que tener una sonrisa en la cara, ser muy sociable con la gente" (Entrevista 4, diciembre de 2016, Bogotá.) 
"Siempre lo primero es el físico, debe tener rostro porque el rostro llama la atención del cliente $y$, pues dependiendo el cliente, tener el cuerpo ya me refiero a lo que es el pene y lo que es la parte trasera es algo que es primordial y la higiene, la higiene del hombre son las cosas más importantes" (Entrevista 8, diciembre de 2016, Bogotá.)

A partir de ello se logra poner en evidencia que el trabajo sexual masculino se traduce en una actividad económica que produce un intercambio de servicios generalmente de tipo sexual o erótico hacia el cuerpo y la expresión de género masculino en palabras de (Hernández \& Espinosa, 2015) esta "se ejerce "libremente" sin coacción ni sometimientos por parte de los implicados, es en este sentido, un intercambio sexual con fines económicos."(p 238), que aún sigue siendo vista por la parte institucional y estatal (en la mayoría de los casos) como un problema de salud pública, ya que debido a las aristas intrínsecas a este fenómeno social y a los contextos en la que se desenvuelve, así como en las prácticas y dinámicas del mismo, se hace evidente el riesgo de propagación de enfermedades de transmisión sexual, entre otros,(Parces ONG, 2016) problemas que permean la realidad social de los sujetos que la ejercen.

\section{Características psicosociales de los trabajadores sexuales masculinos, una reconceptualización de apoyo, necesidad y objeto}

Teniendo como punto de referencia que las características psicosociales se estructuran en el sujeto por un componente afectivo-cognitivo, que a través de la construcción histórico social permean el actuar y sentir del individuo(Salmerón, 2011). Se ha logrado comprender a través de los relatos de los trabajadores sexuales masculinos, que estos conciben a esta estructura como relaciones de apoyo de tipo emocional y económico. Estas repercuten de manera significativa en el desarrollo de sus narrativas que aluden según sus prácticas y experiencias vividas, dada la interacción de los mismo con personas que reconocen como pilares fundamentales de una relación directa de "apoyo" y motivación, evidenciando así en primera medida a la familia de origen (o consanguínea), a los amigos y la pareja(Holgin, 2012).

“Tener una familia con quien apoyarme, en quien apoyarme, heee tener amigos... tener compañeros! amigos no, tener compañeros en la vida con los que uno vive experiencias" (Entrevista 8, enero de 2017, Bogotá.)

Pero los cuales se desgastan con el tiempo, debido a que la relación de "apoyo" con los sujetos se debe fundamentar en tres principios para lograr ser verdaderamente significativo para éstos; "respeto, confianza y tiempo de permanencia”. (Barrera y Fuquene, 2018), esto repercute de manera directa en el conocimiento del ejercicio de la actividad económica del trabajo sexual para con su familia de origen, amigos y la pareja (en los casos donde existe una) e.

Esto debido a que el sexo servicio es visualizado como un elemento de toma de decisiones $y$ de intimidad que inscribe el sujeto, e, (Madero, 2012), y del cual por temor a reprimendas o prejuicios morales, inhibe su conocimiento generalmente a su familia de origen, , hecho que la relega a un plano secundario. A partir de ello se da mayor estabilidad e importancia en términos de apoyo y reciprocidad a la relación establecida con los amigos y la pareja (en los casos en la que existe), (Barrera y Fuquene, 2018).

"Se basa mucho en el respeto, en la confianza, el caso que a mí me rige a mi mundo como tal eso ya hoy en día es como un tabú, para mí no es algo que un pueda presenciar o vivir de buena manera" (Entrevista 1, diciembre de 2016, Bogotá.)

"No nada [...] pues porque es algo personalmente que es para mí o sea es donde yo trabajo y o sea como y creo que a nadie le debe importar pues dónde yo camello" (Entrevista 2, diciembre de 2016, Bogotá.) 
Evidenciando que pese a que se tiende a ocultar el ejercicio de esta actividad económica a la familia, inhibiendo aspectos fundamentales descritos con anterioridad, para establecer relaciones de apoyo, esta es vista en la mayoría de los casos como un eje fundamental de la vida y la realidad de los sujetos, (Zaro, 2008) ya que para algunos esta es o fue motivante, por carencias económicas internas o por abandono dada la orientación sexual o identidad de género en la historia de vida de los sujetos, relegando una firme trascendencia en, independientemente haya o no nexos con esta (Tirado, 2013). Aunque en algunos casos esta se transforma o se relega ya sea a amigos o pareja o al sujeto mismo.

"Pues...jmi familia no! Mi familia [...] a ellos yo les ayudo, pero no saben de dónde saco el dinero, piensan que estoy trabajando de mesonero [...] y pues mis amigos sí, porque las amistades en las que me rodeo ahorita son las mismas de este trabajo (Entrevista 4, diciembre de 2016, Bogotá.)

Trayendo así a colación el componente "social" de las características psicosociales, ya que estas aluden a las relaciones, normas y contextos en los cuales el individuo construye arquetipos sociales (Salmerón, 2011), con lo que el hecho de ejercer el trabajo sexual masculino, traería en sí mismo ciertas repercusiones y realidades que se establecen en la construcción del individuo, sus relaciones y su discurso.

"Eee pues yo desde hace varios años aproximadamente como unos 4 años, 3 años empecé teniendo sexo casual y encuentros casuales, como lo ratifico nuevamente por placer, pero teniendo en cuenta siempre varios riesgos y límites" (Entrevista 1, diciembre de 2016, Bogotá.)

Los trabajadores sexuales conciben que el hecho de ejercer esta actividad económica nace por motivaciones relacionadas en la mayoría de los casos por una necesidad estrictamente económica (dadas principalmente por carencias económicas dentro del núcleo familiar procedente), o por el hecho de satisfacer placeres y deseos sexuales en el cual el cuerpo es visto como un objeto de deseo por el cliente (Osorio et al., 2006). Motivaciones que trascienden el tiempo en cuanto a el ejercicio del trabajo sexual masculino como una fuente fija de ingresos, que obliga al individuo a erotizar y disfrutar de sus prácticas laborales, ya que estas en sí mismas son actos producto de decisiones de forma voluntaria del actor social independientemente del estatus o la edad de los mismos (Salmerón, Ballester, Gil, y Ruiz, 2010).

"Uno trata de convencer al cliente para uno salir complacido, pero con lo que uno necesita que es el dinero" (Entrevista 4, diciembre de 2016, Bogotá.)

"Como una especie de objeto fetichista porque eso es más lo que vendo yo como fetiches, entonces lo veo más como algo muy sexual o sea lo veo más sexy, provocativo, morboso, así lo veo" (Entrevista 6, diciembre de 2016, Bogotá).

Construyendo así una relación entre necesidad económica, placer, toma de decisiones. corporalidad (expresión y erotización de género masculino), que forman una brecha paralela entre la utilidad y el establecer relaciones de apoyo que en sí mismas permean las características y las realidades de un estilo de vida, (Holgin, 2012) que acogen (en su mayoría) los actores sociales, independientemente de su posición económica, nivel de estudios, o residencia.

\section{Prácticas sociales, realidades en un ejercicio de actividad económica}

Teniendo como punto de partida que las prácticas sociales hacen referencia a todas aquellas acciones en el "quehacer de los sujetos en el mundo social, bien sea como interacción, transformación o actividad" (Betancourt et al., 2015, 
p. 20) ), en las que según motivaciones y toma de decisiones en el individuo, "ocurren dentro del marco de los posibles que tienen lugar en el interior de las estructuras, que a su vez guardan coherencia con los principios generadores y constitutivos del hábitos; es decir, con las estructuras sociales"(Betancourt et al., 2015, p. 20), se logra evidenciar, que estas son reflejadas en la cotidianidad de los Trabajadores Sexuales Masculinos, en relaciones interdependientes a las realidades sociales de los individuos, que son en cierta medida determinadas por el estatus en el cual se ejerce esta actividad, económica, sitio y tipo de cliente.

Por tanto, estos asumen y reconocen los riesgos y ventajas que acarrea el hecho de replicar este tipo de prácticas sociales, que en sí mismas propiciaron según las estructuras sociales heteronormativas colombianas, realidades de violencia, discriminación, prejuicio, persecución, problemáticas en cuanto a atención y determinantes sociales en salud, entre otros (Zaro, 2008) Como también se resalta que el incentivo económico juega un papel determinante, en cuanto a la construcción de estos patrones conductuales en el entorno, en el que el sitio de ejercicio de este según el estatus, así como también el tipo de cliente, (que es visto como en muchos casos, un proveedor de lucro e influencia), propician la constitución misma de estructuras sociodemográficas o lugar, y a su vez las prácticas intrínsecas en esta (Parces ONG, 2016).

"Pues desde mi parte de la webcam yo creo que sería como que eee hay gente como que te graba o sea hay clientes que te pagan un show, pero a la misma vez te están grabando y pueden subir eso a internet" (Entrevista 7, enero de 2017, Bogotá.)

Estas estructuras sociales, como ya se había mencionada con anterioridad están permeadas por violencias, las cuales se establecen en lugares propios y estarían a su vez relacionados por condiciones derivadas del entorno. Cosa que Parces
ONG ratifica en su informe de derechos del observatorio de trabajo sexual "Ley entre comillas", en el que se especifica la relación de ciertos actores sociales que propongan prácticas de discriminación y rechazo, de tipo verbal y físico, traducidas en experiencias de violencia por parte de "civiles, clientes y policías" (Parces ONG, 2016, p. 20). Las que a su vez son reconocidas en los discursos de los trabajadores sexuales masculinos, que refieren, además, la segregación de estos debido a sus prácticas y orientaciones sexuales.

"Pues por policías nos agrede cuando él quiere nos corre, no nos deja trabajar y pues los clientes también son malos a veces después que ellos terminan o algo no caen en cuenta en lo que hicieron de acostarse con otro hombre y pues ahí le agarran represalias con uno y lo insultan o lo te puntean o algo" (Entrevista 3, diciembre de 2016, Bogotá).

"Los maltratos de los hombres hacia uno (...) porque si como uno a veces se va con clientes bien, uno no sabe por ejemplo en las noches con qué clase de persona se monte (...) Una vez un cliente en Cúcuta me agredió, me dejo tres meses en muletas, me torció la rótula o sea... (silencio) son muchas cosas que lo cansan a uno, la misma pelea entre los compañeros de trabajo, el peligro, la denigración de la gente, porque cualquiera lo ve a uno ahí parado y piensan que uno es un ladrón, piensan que uno es ratero y pues si hay personas así pero no todos somos iguales" (Entrevista 5, diciembre de 2016, Bogotá).

Ahora bien, las prácticas sexuales como expresión máxima de las prácticas sociales, de los trabajadores sexuales masculinos en su entorno, entendió a estas como "los espacios de fuga, ruptura y resistencia dentro del marco de una estructura social claramente heteronormativa" (Betancur, Ariza, Sánchez, 2015, p. 22), en que el fin último es obtener placer por medio de estímulos 
visuales y físicos (entre otras) en el individuo.

Según las vivencias y el discurso de los trabajadores sexuales masculinos, relegan una gran importancia a r la expresión de género masculina y espacios de riesgos en términos de salud sexual, en aspectos tales como violencias, consumo de sustancias psicoactivas, entre otras, que determinan en el ejercicio de esta actividad económica una toma de decisiones subordinada por la conciencia y el establecimiento de límites en el sujeto ya que para muchos esta no es su única fuente de ingresos, pero que incide de manera directa su proyecto de vida.

"Tal vez que lo quieren someter a uno como a cosas que ellos desean como vamos allí y yo no quiero, pero o cómo te quedas y no te vas de acá o algo, pero yo creo eso depende si uno se deja, si uno es vulnerable y se deja manipular" (Entrevista 1, diciembre de 2016, Bogotá).

"Yo tengo varias fuentes de ingresos, así como puedo trabajar de, así como soy trabajador sexual soy estilista y maquillador" (Entrevista 8, enero de 2017, Bogotá).

"No, no es mi única fuente de ingreso pues yo trabajo con mis velitas, pero pues está muy pesado mis velas, entonces es como mi ruta de escape, en este momento es mi ruta de escape la prostitución". (Entrevista 9, enero de 2017, Bogotá).

La relación estatus-tipo de cliente-lugar, determinarían las prácticas sexuales y el nivel de incentivo económico estipulado en un mutuo acuerdo, por ello es que esta en sí misma instaura una tendencia que denotaban en el sujeto múltiples reacciones en cuanto al ejercicio mismo del trabajo sexual masculino(Barrera y Fuquene, 2018). Prácticas de las cuales se destacan la penetración anal, sexo oral, shows estéticos de sadomasoquismo, y en general el fetichismo, con el fin de atraer y mantener con regularidad una mayor cantidad de clientes (Holgin, 2012). Esto según el estilo de vida, necesidad, placer, los motivos para y porque, tiempo dedicado, continuidad, entre otros determinantes, que estipula el trabajador sexual masculino, para el desenvolvimiento de esta actividad económica, y las prácticas sexuales arraigadas a éste.

Se pone en evidencia que, según el proyecto de vida de los trabajadores sexuales masculinos, estos tienden a transitar por los estatus e incluso por el género, construyendo relaciones que permean la identidad, la corporalidad y el territorio. ya que este cambia su expresión de género, (López, 2010) (sin sentirse identificado con este o puesto) o en la que ejercen como modelos webcam y como Scort a la vez, con el fin de incrementar su nivel de ingresos y mantener un estilo de vida propio.

"Pues ahorita en estos momentos salgo de lunes a lunes, lo que es de lunes a jueves trabajo en el centro en terraza, en lo que es en el día en la tarde y viernes sábados y domingos trabajo de 9 de la noche a 6 de la mañana, toda la noche por aquí por chapinero, por lo que me toca pagar arriendo diario y eso" (Entrevista 5, diciembre de 2016, Bogotá).

Las prácticas sexuales descritas a través de los discursos de las personas que ejercen el trabajo sexual masculino, se harían evidentes determinantes en cuanto a la salud traducidas en factores de riesgo y protectores para, que inciden en el padecimiento del VIH (Virus de Inmunodeficiencia Humana) o de alguna clase de enfermedad de transmisión sexual, ya que pesa que la mayoría expresa que no consume sustancias psicoactivas con los clientes, (Tirado, 2014) estos las consumen esporádicamente y asimismo ponen en evidencia que los mismos clientes en algunos casos les exigen estar bajo estos efecto para acompañarlos a ellos durante la práctica sexual, también dependiendo de la misma y de muchos fetiches bajo los cuales se contratan los servicios, se encuentra dependiendo del estatus la mayoría de sitios donde se prestan estos no cuentan con las condiciones óp- 
timas y dependiendo del mismo, se ratifican prácticas de riesgo en las que solo se tiene en cuenta el uso y la exigencia del preservativo para evitar ITS (Madero, 2012).

"Normalmente cuando yo hablo con mis clientes hay muchos que no les gusta tener sexo sin condón ellos yo siempre aclaro que siempre uso condón y soy yo el que siempre ha tomado la iniciativa" (Entrevista 8, enero de 2017, Bogotá).

También es necesario aclarar que, en cuanto a factores de riesgo para contraer VIH, los trabajadores sexuales masculinos abordados, conocían cuáles eran estos, pero se pone en evidencia que no se lleva con control regular en cuanto a prueba diagnóstica de tipo ideal (cada tres meses), ya que se desconocen los sitios para realizarse la misma. Así como que es tendencia que estos porten y exijan el uso de preservativo al cliente, puesto que este comúnmente pide el no uso de este o por temor a que esté roto o en malas condiciones.(Salmerón et al., 2010) Ante ello estos afirman atender según estatus a un promedio de 1 a 6 clientes por día, o de 2 a 10 shows (en el caso de modelos webcam), obteniendo ingresos diarios de 0 a $\$ 600.000$, porcentajes que varían, según factores medioambientales, económicos, etc.

"Mensual se pueden ganar una persona que trabaje espontáneamente puede ganar dos millones cómodamente y una persona que ya quiera, una persona que quiera ya la profesión como tal, como tal estando constante, en un mes se puede hacer hasta más de seis, siete millones" (Entrevista 10, enero de 2017, Bogotá.)

"Hacer sexo oral, por medio del contacto de saliva por medio de contacto de sangre, por medio de la penetración sin protección (...) sii (...) casi 7 o a veces dejo pasar 8 meses y así". (Entrevista 1, diciembre de 2016, Bogotá.)
Las prácticas sociales y sexuales, propias de un trabajador sexual masculino, serían entonces inter dependientes de la construcción histórica social del sujeto, el estatus, lugar y tipo de cliente, generando dinámicas o constructos sociales que permean el desenvolvimiento de esta actividad económica. Por tanto, los factores de riesgo para contraer algún tipo de enfermedad de transmisión sexual, se ven reflejadas como un riesgo continuo $y$, consciente en el desenvolvimiento del sexo servicio y en la que de igual manera el sujeto constituye factores protectores (según el estatus), (Tirado, 2014), tales como el uso y porte del preservativo, lubricante, entre otros.

Estas prácticas se inscriben en el sexo servidor, a partir de aspectos tales como motivantes personales, tipo de cliente y necesidad de lucro para el desenvolvimiento de fetiches, consumo de sustancias psicoactivas, entre otras. Que se hacen evidentes en las narrativas y experiencias vividas de los trabajadores sexuales masculinos.

"Enfermarse de VIH, pero eso no lo puede evitar cuidándose mucho $\mathrm{y}$ otros riesgos es que en cualquier momento usted puede perder la vida y nadie va a llorar por usted, ni nadie va a evitarlo porque usted está en la calle y se mete con un hombre y va y uno se monta un carro uno no sabe quién es esa persona, qué esté pensando, que como esté entonces uno tiene el riesgo de perder la vida" (Entrevista 1, diciembre de 2016, Bogotá).

\section{Conclusiones}

La personas que ejerce el trabajo sexual masculino como actividad económica en varios sectores de Bogotá, y pertenecientes a varios "status", poseen características, que a su vez son un producto derivado de procesos histórico sociales que permean el desenvolvimiento de ciertas prácticas sociales y a su vez sexuales que trascienden 
en la construcción misma del sujeto, y de la identidad con la que este se relaciona con el ejercicio de la misma, siendo así consciente de los diversos riesgos, beneficios y problemáticas arraigadas a la realidad de este.

Las características psicosociales de los trabajadores sexuales masculinos, se traducen en un constructo socio histórico-cultural del sujeto, en el que el entorno de socialización primaria (familia), configura una serie de cualidades y prácticas en el sujeto, por tanto es que en la mayoría el choque del afrontamiento de su orientación sexual, se toma como punto clave de análisis para la conformación y definición del establecimiento de relaciones de apoyo, donde se entretejen principios de respeto, confianza y tiempo, en el que los amigos y la pareja se presentan como refugio y consejo emocional y aunque se destaca la importancia de la familia esta es vista mayormente como proveedora económica y responsabilidad del sujeto, pero en la que en la mayoría de los casos se invisibiliza por temor a rechazo y discriminación, el ejercicio del trabajo sexual como actividad económica.

Las prácticas sociales se configuran en los trabajadores sexuales masculinos, como un reflejo de sus características psicosociales, pero construidas en una relación entorno-status y tipo de cliente, por lo que estos adaptan y replican características físicas que en sí mismas se predeterminan según el contexto, en el que se a su vez dan cabida a prácticas sexuales determinadas que de manera consciente y voluntaria el actor social, en la que en algunos acasos se transita de manera momentánea el género masculino con el femenino sin que este se sienta identificado, por lo que se brinda según la necesidad de ganancia económica, tipo de clientes foco y tipo de mercado, acordonado y ofreciendo cierto de tipos de servicios sexuales y estéticos en el que se trata que tanto el cliente como quien los brinda salgan complacidos, tales como, penetración anal, sexo oral, fetiches, sadomasoquismo, bailes eróticos, etc., siendo la expresión de género masculina un factor clave para ello.
Por otra parte muchas de estas prácticas sexuales permean una serie de factores de riesgos en términos de determinantes sociales en la salud para contraer el VIH o alguna enfermedad de transmisión sexual, en la media en la que se evidencia que muchos lugares en los que se practica no cuentan con las condiciones sanitarias óptimas, así como el consumo de sustancias psicoactivas entre otras, aunque cabe recalcar que existe conciencia acerca de medios de transmisión del VIH y las ETS más comunes y se recalca el uso del condón como principal medida de protección.

\section{Referencias}

Barrera, J., \& Fuquene, J. (2018). “YYo también puteo!". Realidades del trabajo sexual masculino en Bogotá D . C . Trabajo Social, 20(2), 157-175. Retrieved from doi: https://doi. org/10.15446/ts.v20n2.74310

Betancourt, C., et al. (2015). Practicas sexuales entre hombres: tensiones entre la prevencion $y$ el deseo en Bogota D.C. Bogota.Estudio exploratorio Informe Final. Bogota: Corporación Red Somos, Secreta de Salud de Bogotá D.C.

Bonilla, E., \& Rodriguez, P. (1997). Mas alla del dilema de los metodos : la investigacion en ciencias sociales. Bogota: Editorial Norma.

Gergen, K. J. (2007). Constructivismo social: aportes para el debate y la práctica. Bogota: Universidad de los Andes, Facultad de Ciencias Sociales.

Hernández, L., \& Espinosa, M. (2015). Trabajo Sexual Masculino. Yo Puto. repensando el Trabajo Sexual Masculino. Genero y Direto, 5(3), 281-312. https://doi.org/10.18351/21797137/ged.2015n1p281-312

Holgin, D. (2012). Los invisibles aproximación al significado de las redes primarias de los hombres que ejercen la prostitución en el parque de Lourdes y terraza Pasteur. Universidad Colegio Mayor de Cundinamarca.

Madero, A. S. (2012). Cuerpos En Venta: Pinguerismo Y Masculinidad Negociada En La Cuba Contemporánea. Nomadas, 26(6), 
167-183.

Mendicoa, G. E. (2003). Sobre tesis y tesistas : lecciones de enseñanza-aprendizaje. Barcelona: Espacio Editorial.

Osorio, L. et al.. (2006). Prostitución masculina: manifestaciones, características y problemas asociados en las localidades de Mártires, Santafe y Teusaquillo de Bogotá D.C. Universidad de la Salle. Universidad de la Salle.

Parces ONG. (2016). Ley entre comillas: informe de derechos del observatorio de trabajo sexual. Bogota: Imagenes y textos S.A.

Salmerón, P. (2011). Perfil Psicosocial de los Trabajadores Masculinos del Sexo. Universidad Jaume. Universidad Jaume.

Salmerón, P., Ballester, R., Gil, D., \& Ruiz, E. (2010). Perfil Sociodemográfico de los trabajadores sexuales: adolescentes y jóvenes en riesgo de exclusión social. INFAD: Revista de Psicología, 20, 759-768.

Tirado, M. (2005). Creencias y comportamientos socio-culturales de jóvenes del género masculino prostituidos homoeróticamente en Bogotá. Revista Colombiana de Soci- ología, 24, 93-113. Retrieved from file://D:/ Downloads/11296-27361-1-PB.pdf

Tirado, M. (2011). EL debate entre Prostitucion y Trabajo Sexual. Una mirada desde lo sociojurídico y la política pública Misael. Revista de Relaciones Internacionales, Estrategia y Seguridad, 6(1), 127-148.

Tirado, M. (2013). Comercio sexual (2nd ed.). Instituto Latinoamericano de Altos EstudiosILAE. Bogotá.

Tirado, M. (2014). El trabajo sexual desde una perspectiva de los derechos humanos: implicaciones del VIH/sida e infecciones de transmisión sexual* Sex work from a human rights perspective: implications of $\mathrm{HIV} /$ AIDS and sex transmitted infections. Civilizar, 14, 97-110.

Zaro, I. (2006). Trabajadores Masculinos Del Sexo: Aproximación a La Prostitución Masculina En Madrid. Fundacion Triangulo Negro.

Zaro, I. (2008). La prostitución masculina. Un Colectivo Oculto Y Vulnerable . Revista d'Estudis de La Violència, 6, 1-18. 